MATEC Web of Conferences 44, 01046 (2016)

DOI: $10.1051 /$ matecconf/20164401046

(C) Owned by the authors, published by EDP Sciences, 2016

\title{
Modelling of technological reliability in traffic logistic networks in urban areas
}

\author{
Reiner Keil ${ }^{1}$, Frank Hedrich ${ }^{2}$, Iveta Kremenova ${ }^{3}$ and Radovan Madlenak ${ }^{3}$, a \\ ${ }^{1}$ Saxon Telecommunications Center, Faculty of Transportation and Traffic sciences, Technical University of Dresden, 01062 Dresden, \\ Germany \\ ${ }^{2}$ Technical University of Dresden, Faculty of Transportation and Traffic sciences, 01062 Dresden, Germany \\ ${ }^{3}$ University of Zilina, The Faculty of Operation and Economics of Transport and Communications, 01026, Zilina, Slovakia
}

\begin{abstract}
The trend of growing parcel numbers with the customer's desire for shorter delivery times as a result of increasing online trade and economic specialization requires an adjustment of transport systems. The share of transportation costs per postal item depends mainly depends on costs for employees and vehicles, in addition are still the costs of processing in the sorting centers. There are only predictions and experiences for the amount of postal item are available for the tour in advance. The difficult planning leads, in addition to the high cost share and the key feature of a high quality of service to a large relevance to the total transportation process. It is presented an analytical model of the transport process. This includes models to forecast the number of postal items, for capacity-oriented planning of tours and a determination of the current vehicle usage and the volume of transported parcels.
\end{abstract}

\section{Introduction}

Scientific based organization of transport processes relies on elaboration of analytic or heuristic models. This paper deduces a model for uniform analysis of traffic organization processes based on traffic theory [4] using the parcel pickup process in urban area for model validation. Operating data cannot be acquired prior to stationary processing in sorting centers [5]. The initial lack of accurate raw data requires statistical data treatment of operating data for tour design. Explicit data on pickup tours, for example utilization of letterboxes or vehicles, is liable to subjective errors, hence constrained in use for modelling purposes.

\section{Basic model for analytic description of transport logistic processes}

Initial step for analytic modelling of transport logistic processes is deduction of fundamental processes based on universal operation process definition used in logistics and telecommunication [4]:

\section{Operate $=$ serving, maintenance and monitoring}

A traffic-logistic process can be described by the following basic processes, which run off at the same time and relatively independently:

- Traffic object input and output, consisting of the traffic process with the characteristic random variables traffic object quantity $X$ and the entrance process with the random variable entrance time $T_{E}$, the exit process is not explicitly regarded,

- Technological process, handling and transportation processes - modelled as serving process,

- Operating disturbance - modelled as a failure process,

- Maintenance - modelled as a recovery process.

Modelling of the afore mentioned processes was carried out using the specific probability model in [4] and can be characterized by the figure 1 .

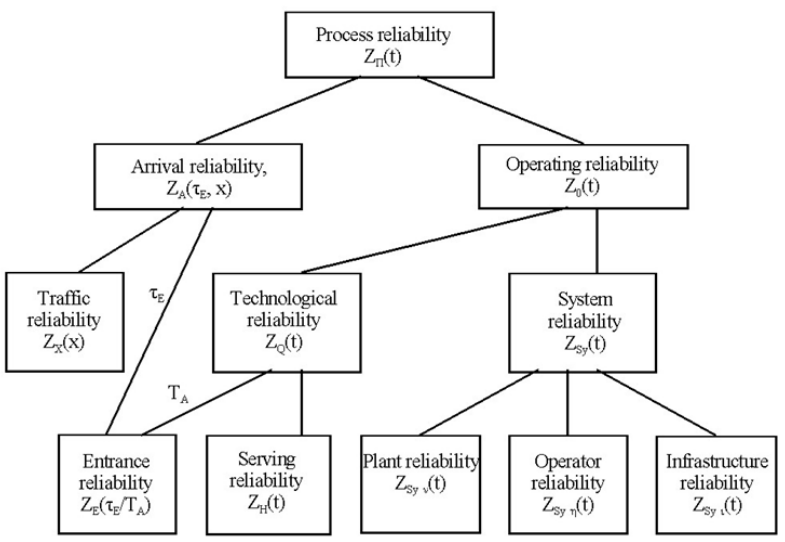

Figure 1. Reliability model

Process reliability $Z_{\Pi}(t)$ is defined as the probability of a system configuration SY using maintenance method IM during the time period $t$ being able to fully serve the amount of traffic objects $X$ at a point in time $T_{E}$.

\footnotetext{
a Corresponding author: radovan.madlenak@fpedas.uniza.sk
} 


$$
Z_{\Pi}(t)=P\left[T_{Q} \mid\left(X \mid T_{E} \wedge S Y \wedge I M\right) \leq t\right]=P\left[T_{Q} \mid\left(X\left|T_{E} \cap T_{\lambda}\right| T_{\mu} \geq t\right) \leq t\right]
$$

Within this model, technological reliability describes the operation reliability $Z_{Q}(t)$ - characterizing the operation process in nodes and edges with the inbound probability $Z_{E}\left(\tau_{E} / \mathrm{T}_{A}\right)$ - characterizing the inbound process by using the inbound point in time $\tau_{E}$ or the arrival interval $\mathrm{T}_{A}$ depending upon operation process.

Utilizing traffic reliability $Z_{X}(x)$, corresponds the traffic objects' distribution function,

$$
Z_{X}(x)=F_{X}(x)
$$

includes the stochastic of the amount of traffic object. In this instance, the traffic objects are given by letters and parcels. Using an Erlang - $\mathrm{k}$ distribution to model the traffic objects quantity [3], the following distribution formula applies

$$
F_{X}(x)=\int_{0}^{x} \frac{\lambda^{k} \xi^{k-1} e^{-\lambda \xi}}{(k-1) !} d \xi
$$

Parameters $\lambda$ and $k$ are calculated using the estimates for expected value and variance

$$
\lambda=\frac{E(X)}{D^{2}(X)}, \quad k=\frac{E^{2}(X)}{D^{2}(X)}
$$

Raw data has to be gathered and edited to determine expected value and variance.

\section{Data collection and preparation}

\subsection{Collection of the amount of traffic objects}

Transport process planning depends on knowledge about estimated amount of traffic objects to ensure high capacity utilization via suitable vehicle selection and adjusted tour planning. On the other hand, backlog must not occur. Those result from a discrepancy between vehicle capacity and current amount of traffic objects. With the complete recording of traffic objects during stationary processing in sorting centers, the distribution function of traffic objects can aid the probability of backlog $P_{R}$.

With calculation utilizing formula (2)

$$
P_{R}=1-F_{X}\left(x=C_{F z g}\right)
$$

Truncate the distribution function on the upper ceiling oft the amount of traffic objects $x_{o}=C_{F z g}$ yields vehicle utilization $\eta_{F z g}$. Truncate in the distribution function within a interval $\alpha \leq u \leq \beta$ are calculated according to [4]

$$
\widetilde{F}_{U}(u)=\frac{F_{U}(u)-F_{U}(\alpha)}{F_{U}(\beta)-F_{U}(\alpha)}
$$

Applying formula (3) with $F_{X}(\alpha=0)=0$ to Erlang - k distribution results

$$
\eta_{F z g}=\tilde{F}(x)=\frac{F_{X}(x)}{F_{X}\left(C_{F z g}\right)}
$$

During stationary processing in outbound hubs, the amount of traffic objects is measured by counting the unique kinds of traffic objects, for example standard letters, large letters, small parcel and packages. Thus said, tour design is primary limited by volume and mass of traffic objects. Acquisition of traffic object data in outbound hubs limits conclusions of tour specific load and ratio of traffic object types. For instance, tours serving mainly letterboxes or mayor parcel customers vary greatly. In addition, the measured amount of traffic objects underlies fluctuations of various causes requiring the tour design and vehicle assignment furthermore to rely on estimates.

\subsection{Time series analysis}

The time sequence of daily traffic objects can mathematically be described as time series with components for trend, business cycle, seasonal and residual figure. In this instance particularly focusing on the estimation of trend and seasonal components.

The trend component reflects long term change of the examined feature discarding short term and small periodic fluctuations. Presuming the trend component as deterministic allows extrapolation of the trend function to forecast. Long term development is crucial for strategic shaping of the transportation system.

Elimination of irrelevant fluctuations and outliers via smoothing is advisable for long term analysis. Smoothing is a piecewise local adjustment replacing the observations with weighted means of the surrounding observed values. The number of included values and their weighting characterize smoothing methods.

Smoothing of letter and parcel time series aims for balancing the influence of business days and decommission days. At the same time a small smoothing period is advantageous for to keep distortions to the subsequent seasonal analysis low. In order to eliminate business day fluctuations at least a one-week period has to be span. For smoothing the method of a moving average is sufficient. By using the actual, the three prior and subsequent values for the smoothing the smoothing method can be formally described as follows:

$$
y_{t}=\frac{1}{7} \sum_{i=-3}^{3} y_{t+i}
$$

To illustrate the moving average smoothing method an extract of the letter time series is shown below.

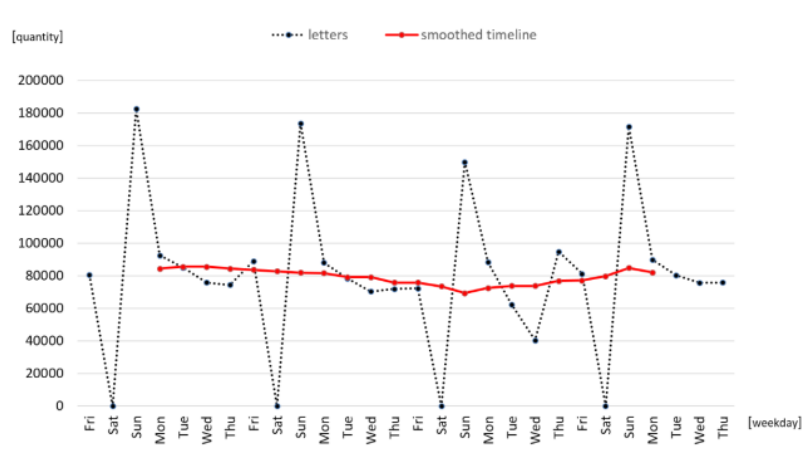

Figure 2. Extract of the letter time series [2] 
Trend regression is used to describe the long term monotone progression of time series in a mathematical manner. With the available raw data, a rather small forecast period, the flat gradient and good regression a linear function of the following type is used to characterize the trend function.

$$
g(t)=a_{0}+a_{1} \cdot t
$$

The parameters $a_{0}$ and $a_{1}$ are estimated using least square minimization. Once the trend function is determined, trend adjustment is carried out by subtracting the trend figure from the initial time series. This allows for further analysis without interference of the trend function. Another periodic fluctuation in quantity of traffic objects occurs at the end of the year. Those fluctuations are equal in strength and depend in the base amount of traffic objects. This insight is incorporated into the regression model by linking the seasonal figure multiplicatively to the base amount, given by the trend function, using a coefficient.

The seasonal component reflects the fluctuations within a year's period. Through sorting the values of the time series by their calendar date enables calculation of the average seasonal figure. Lacking correlation between calendar date and weekdays furthermore independence of seasonal figure and long term development allows to using the smoothed, detrended time series. Due to the complex nature of the seasonal figure a five zone spline was used to capture the specific regression functions individually providing seamless transitions between spline zones. Figure 3 illustrated the seasonal figure and upon based spline modelling of the letter time series.

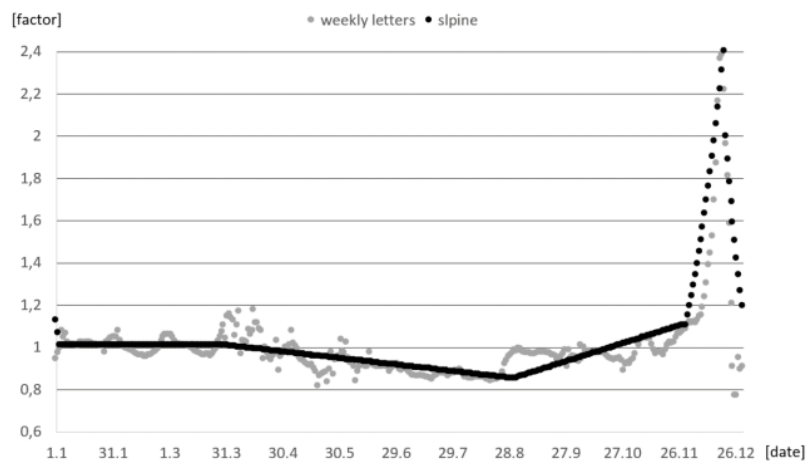

Figure 3. Seasonal figure of letter time series [2]

\subsection{Weekday factor analysis}

Calculation of weekday coefficients is carried out through the ratios of daily quantity of traffic object compared to the weekly moving average. Furthermore, variance analysis is used to examine the separation of weekdays to each other. All values without processed traffic objects, e.g. holydays, are excluded leaving the letter final day coefficients below:
Table 1. Final letter day coefficients [2].

\begin{tabular}{|c|c|c|c|c|}
\hline & Mon & Tue & Wed, Thu, Fri & Sun \\
\hline Day coefficient & 1,087 & 0,986 & 0,931 & 2,262 \\
\hline
\end{tabular}

\subsection{Transport volume enquiry}

The current capacity planning acts on the judgement of the responsible transport planner with circumstantial feedback regarding capacity shortage or lack of service quality by carriers and customers. Ideally vehicle deployment and provided transport capacity with buffer should comply with the expected amount of traffic units. Approaching the coupling of transport volume and quantity of traffic objects through a mean traffic object size is not sufficient given that:

- lack of numerical acquisition of transported letters and parcels in vehicles,

- volume of letters and parcels varies greatly,

- lack of knowledge about share between investigated area and covered total influence area,

- lack of knowledge about share between combined letter and parcel transportation compared to covered total influence area,

A survey of the transported letters and parcels in vehicles serving within the investigation area and the knowledge enable prognosis of the expected transport volume [6].

\subsection{Load state classification}

Connecting transport planning to extrapolated transport volume leads to a variety of loads of the transport system. The introduction of discrete load states can minimize the transport planning effort. Further advantages are:

- ability to forecast due to connection between tour planning and extrapolated transport volume,

- reduction in planning effort through pooling of similar load states,

- stable transport schedules through reuse of planned tours for similar transport loads,

- estimation of consignment load and transportation load of accumulative haul tours through linkage to transport volume aiding planning process of alternative transportation concepts,

- short reaction time to adopt fluctuations in transport volume via premade tour plans.

In contrast to commonly used approach for capacity planning are improvements by linking vehicle assignment and operating data based extrapolation of transport volume likely. Figure 4 presents 12 load states with their respective transport volume and occurrence within a year. The classification of those 12 load states yields the needed number of vehicles. 


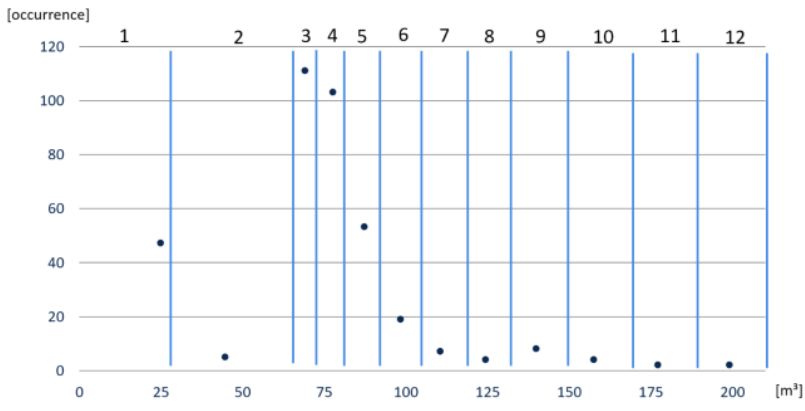

Figure 4. Classification of load states [2]

\subsection{Localization of transport volume}

Final data acquisition step is assignment of transported transport volume to individual locations, e.g. letterboxes, stores or customers. Attention is specially paid to letterboxes due to potentially multiple approaches daily. Localization utilizes geographic coordinates of corresponding address data. Particular urban environments with limited size allow for the simplification of the geographic coordinate system as orthogonal.

\section{Tour planning}

\subsection{Clustering of service area}

After gathering and preparing all planning data, tour planning can be executed. Beginning with the clustering of the service area to allow for individual tour planning within the clusters. In this case the investigation area spreads $500 \mathrm{~km}^{2}$ including 589 relevant locations. Locations concentrate in the center of the investigation area. Clustering is based on the spatial separation of the locations.

\subsection{Calculation of travelled distance}

Calculation of total driven distance distinguishes two types of tours; direct transport for locations with high, vehicle filling transport volume and collection tours with several locations to fill up vehicle capacity. Further investigation additionally evaluated the option of transshipment. Distance calculation for direct transport, routes between terminal and hub likewise route between hub and cluster uses beeline distance and infrastructure based detour factor. Distance travelled within the clusters is based on analogy to Travelling Salesman Problem [1] using regression of solved TSP samples between location count and saved mileage in comparison to a star network. Infrastructure is also represented using detour factors.

\subsection{Travelled distance of load states}

Calculation of distance driven is based on the clustering of locations. The leg between starting point and the first location is called pass way. By estimating the distance travelled within clusters based on analogy to the
Travelling Salesman Problem, the estimated distance travelled is independent of the chosen locations to anchor the pass way. This is also advantageous for the calculation of the total travelled distance consisting of circular tour within clusters and the pass way because different start points for the pass way can comparably be evaluated. Minimizing total distance, the nearest location to a start point within a cluster is chosen figure 5 illustrated the afore mentioned by means of two different start points as well as a circular tour within the cluster. Not changing the result of the TSP round tour estimation, different start points with corresponding path optimized cluster locations yield identical TSP estimates. The difference among both cases being the difference in pass way distance (blue and red line). The total distance driven for collection tours consists of the circle tour estimate and two times the pass way for approach and return. For direct transport, the circle tour is dispensed, leaving two times the pass way distance.

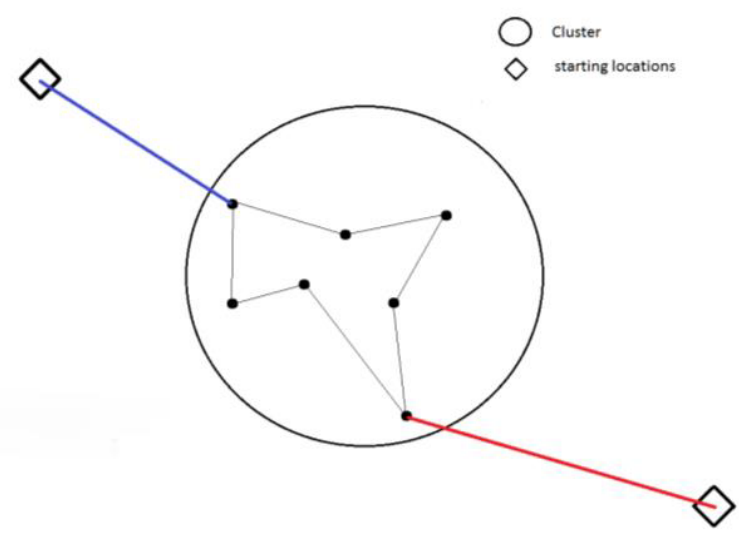

Figure 5. Pass ways of different start points [2]

Distance travelled of each load state is based on three calculated load states and uses interpolation for residual load states. For vans, vehicles mass not exceeding 3500 $\mathrm{kg}$, correlation between transport volume and travelled distance is presented in figure 6 .

Technological reliability of the transport process's finishing time requires the assumption of independent singular trips across the product over transportation durations distribution function of each tour. In this context applies:

$$
Z_{Q}\left(t_{S}\right)=\prod_{k} Z_{Q_{k}}\left(t_{S_{k}}\right)=\prod_{k} F_{T_{T_{l}}}\left(t_{S_{k}}\right)
$$

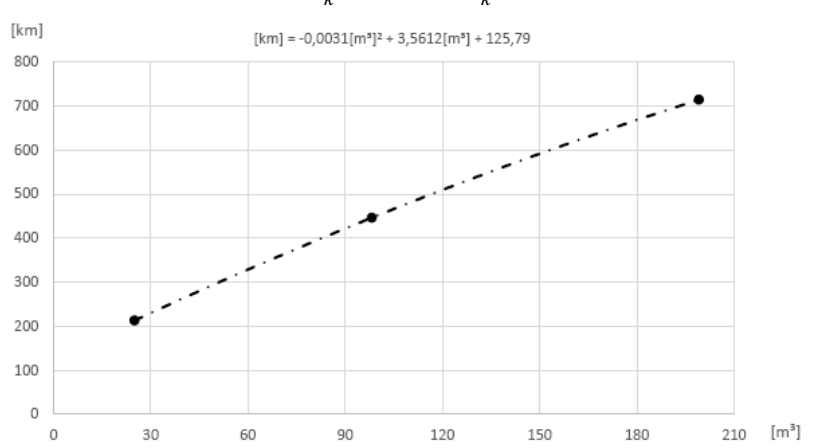

Figure 6. Distance travelled in conjunction with transport volume [2] 


\section{Conclusion}

The introduced technique enables to determine traffic cost for letter and parcel transportation of postal carriers in urban areas. Insufficient data for planning of the initial transportation process - collection of mail and parcel with transportation to sorting centers - and restriction to subjective measurements result in low data quality. The lack in data quality can be compensated through systematic application of mathematical statistical methods.

Time series analysis of processed letters and parcels allows for to forecast the transport volume and promises benefits for capacity planning in sorting centers. Knowledge of estimated parcels and letters allows to adjust the deployment of personnel and set the start of stationary processing. In reverse uncritical processing load allows later start of stationary processing widening the time frame for parcel and letter transportation. Both business areas interact and leave potential for improvement by integrating transportation planning and processing planning.

\section{References}

1. W. Domschke, A. Drexel, Logistik: Rundreisen und Touren. (München: R. Oldenburg Verlag, 1997)

2. F. Hedrich, Modellierung der technologischen Zuverlässigkeit in Verkehrsnetzen der stofflichen Kommunikation. (Dresden, Germany. Technische Univeresität, 2015)

3. R. Keil, Beschreibung des Zeitverhaltens informationslogistischer Prozesse, (Schriftenreihe des Sächsischen Telekommunikationszentrums, 2/2006, Dresden, 2006)

4. R. Keil, Analytische Ansätze zur Modellierung verkehrslogistischer Prozesse - Teil 1 Modellierung (Schriftenreihe des Sächsischen Telekommunikationszentrums, 11/2011, Dresden, 2011)

5. T. Corejova, E. Imriskova, Convergence at the postal market. Eksploatacja i Niezawodnosc. Vol. 39/3, p. 74-76, 2008

6. L. Madleňáková, Layer model of the postal system. (University of Zilina, Zilina, 2013) 